\title{
CONTINUOUS SELECTIONS OF SOLUTION SETS TO EVOLUTION EQUATIONS
}

\author{
VASILE STAICU
}

(Communicated by Kenneth R. Meyer)

\begin{abstract}
We prove the existence of a continuous selection of the multivalued map $\xi \rightarrow \mathscr{T}(\xi)$, where $\mathscr{T}(\xi)$ is the set of all weak (resp. mild) solutions of the Cauchy problem

$$
\dot{x}(t) \in A x(t)+F(t, x(t)), \quad x(0)=\xi,
$$

assuming that $F$ is Lipschitzian with respect to $x$ and $-A$ is a maximal monotone map (resp. $A$ is the infinitesimal generator of a $C_{0}$-semigroup). We also establish an analog of Michael's theorem for the solution sets of the Cauchy problem $\dot{x}(t) \in F(t, x(t)), x(0)=\xi$.
\end{abstract}

\section{INTRODUCTION AND PRELIMINARIES}

The existence of a continuous map $\xi \rightarrow x_{\xi}$ such that $x_{\xi}$ is a solution of the Cauchy problem

$$
\dot{x} \in F(t, x), \quad x(0)=\xi,
$$

where $F$ is Lipschitzian with respect to $x$, was proved first by Cellina in [5], under the assumption that the values of $F$ are nonempty compact sets contained in a bounded subset of $R^{n}$. The case in which $F$ takes nonempty closed values in $R^{n}$ was considered in [6]. Some extensions to Lipschitzian $F$, with nonempty closed values, in a separable Banach space $X$ were treated in $[3,7]$.

The purpose of this paper is to give two similar results for the Cauchy problem

$$
\dot{x}(t) \in A x(t)+F(t, x(t)), \quad x(0)=\xi,
$$

where $F$ is Lipschitzian in $x$, with nonempty closed values and $A$ satisfies assumptions of the type:

(a) $A=-B$ where $B$ is a maximal monotone map on a separable Hilbert space; and

(b) $A$ is the infinitesimal generator of $C_{0}$-semigroup on a separable Banach space.

Received by the editors April 8, 1990.

1980 Mathematics Subject Classification (1985 Revision). Primary 34A60, 49A50, 54C65. 
Indeed, under suitable assumptions on $F$, if $A$ satisfies (a) (resp. (b)), we prove the existence of a continuous selection of the multivalued map $\xi \rightarrow \mathscr{T}(\xi)$, where $\mathscr{T}(\xi)$ is the set of all weak (resp. mild) solutions of (1.2). To do this we adapt the construction in [7] based on Filippov's approach [9]. We also establish an analog of Michael's theorem [12] for the solution sets of the Cauchy problem (1.1). For properties of the set of solutions of $(1.2)$ see also [10,13].

Let $T>0, I=[0, T]$ and denote by $\mathscr{L}$ the $\sigma$-algebra of all Lebesgue measurable subsets of $I$. Let $X$ be a real separable Banach space with the norm $\|\cdot\|$. Denote by $2^{X}$ the family of all nonempty subsets of $X$ and by $\mathscr{B}(X)$ the family of all Borel subsets of $X$. For any subset $A \subset X$, we denote by $\operatorname{cl} A$ the closure of $A$ and if $A \in 2^{X}$ and $x \in X$ we set $d(x, A)=$ $\inf \{\|x-y\|: y \in A\}$. Furthermore for two closed bounded nonempty subsets $A, B$ of $X$, we denote by $h(A, B)$ the Hausdorff distance from $A$ to $B$, that is, $h(A, B)=\max \{\sup \{d(x, B): x \in A\}, \sup \{d(y, A): y \in B\}\}$.

By $C(I, X)$ (resp. $L^{1}(I, X)$ ) we mean the Banach space of all continuous (resp. Bochner integrable) functions $x: I \rightarrow X$ endowed with norm $\|x\|_{\infty}=$ $\sup \{\|x(t)\|: t \in I\} \quad\left(\right.$ resp. $\left.\|x\|_{1}=\int_{0}^{T}\|x(t)\| d t\right) . A C(I, X)$ stands for the Banach space of all absolutely continuous functions $x: I \rightarrow X$ with the norm $\left.\|x\|_{A C}=\|x(0)\|+\|\dot{x}\|_{1}\right)$.

Recall that a subset $K$ of $L^{1}(I, X)$ is said to be decomposable [11] if for every $u, v \in K$ and $A \in \mathscr{L}$, we have $u \chi_{A}+v \chi_{I \backslash A} \in K$, where $\chi_{A}$ stands for the characteristic function of $A$. We denote by $\mathscr{D}$ the family of all decomposable closed nonempty subsets of $L^{1}(I, X)$.

Let $S$ be a separable metric space and let $\mathscr{A}$ be a $\sigma$-algebra of subsets of $S$. A multivalued map $G: S \rightarrow 2^{X}$ is said to be lower semicontinuous (1.s.c.) if for every closed subset $C$ of $X$ the set $\{s \in S: G(s) \subset C\}$ is closed in $S$. The map $G$ is said to be $\mathscr{A}$-measurable if for every closed subset $C$ of $X$, we have that $\{s \in S: G(s) \cap C \neq \varnothing\} \in \mathscr{A}$. A function $g: S \rightarrow X$ such that $g(s) \in G(s)$ for all $s \in S$ is called a selection of $G(\cdot)$.

We agree to say that a multifunction $F: I \times X \rightarrow 2^{X}$ satisfies $(\mathrm{H})$ if $F$ takes nonempty closed bounded values, and

$\left(\mathrm{H}_{1}\right) F$ is $\mathscr{L} \otimes \mathscr{B}(X)$-measurable;

$\left(\mathrm{H}_{2}\right)$ there exists $k \in L^{1}(I, R)$ such that $h(F(t, x), F(t, y)) \leq k(t)\|x-y\|$, for all $x, y \in X$, a.e. in $I$;

$\left(\mathrm{H}_{3}\right)$ there exists $\beta \in L^{1}(I, R)$ such that $d(0, F(t, 0)) \leq \beta(t), t \in I$ a.e.

The following two lemmas are used in the sequel:

Lemma 1.1 [7, Proposition 2.1]. Let $F^{*}: I \times S \rightarrow 2^{X}$ be $\mathscr{L} \otimes \mathscr{B}(S)$-measurable with nonempty closed values, and let $F^{*}(t, \cdot)$ be l.s.c. for each $t \in I$. Then the map $\xi \rightarrow G_{F^{*}}(\xi)$ given by

$$
G_{F^{*}}(\xi)=\left\{v \in L^{1}(I, X): v(t) \in F^{*}(t, \xi) \text { a.e. in } I\right\}
$$


is l.s.c. from $S$ into $\mathscr{D}$ if and only if there exists a continuous map $\beta: S \rightarrow$ $L^{1}(I, R)$ such that for all $\xi \in S$, we have $d(0, F(t, \xi)) \leq \beta(\xi)(t), t \in I$ a.e.

Lemma 1.2 [7, Proposition 2.2]. Let $G: S \rightarrow \mathscr{D}$ be a l.s.c. multifunction, and let $\varphi: S \rightarrow L^{1}(I, X)$ and $\psi: S \rightarrow L^{1}(I, R)$ be continuous maps. If for every $\xi \in S$ the set

$$
H(\xi)=\operatorname{cl}\{v \in G(\xi):\|v(t)-\varphi(\xi)(t)\|<\psi(\xi)(t), \text { a.e. in } I\}
$$

is nonempty then the map $H: S \rightarrow \mathscr{D}$ defined by (1.3) admits a continuous selection.

The second lemma is a direct consequence of Proposition 4 and Theorem 3 in [2].

In $\S 2$ we study the Cauchy problem (1.2) in the case $A$ satisfies (a). The case of $A$ satisfying (b) is considered in $\S 3$. In the last section we prove an extension result of Michael's type for the solution sets of (1.1).

\section{2. $A=-B$ WHERE $B$ IS A MAXIMAL MONOTONE MAP}

In this section $X$ is a real separable Hilbert space with scalar product $\langle\cdot, \cdot\rangle$ and norm $\|\cdot\| . B$ is a maximal monotone map on $X$ with domain $D(B)=$ $\{x \in X: B x \neq \varnothing\}$. Recall that $B$ is said to be maximal monotone on $X$ if:

(i) for all $x_{1}, x_{2} \in D(B)$ and all $y_{1} \in B x_{1}, y_{2} \in B x_{2}$, we have $\left\langle y_{1}-\right.$ $\left.y_{2}, x_{1}-x_{2}\right\rangle \geq 0 ;$ and

(ii) for every $y \in X$, there exists $x \in D(B)$ such that $x+B x=y$.

As is well known [4] $\operatorname{cl} D(B)$ is convex, and for each $x \in D(B)$, the set $B x$ is closed and convex.

For $\xi \in \operatorname{cl} D(B)$ and $f \in L^{1}(I, X)$, we consider the Cauchy problem

$$
\dot{x}(t) \in-B x(t)+f(t), \quad x(0)=\xi .
$$

Definition 2.1. A function $x: I \rightarrow X$ is called a strong solution of the Cauchy problem $\left(P_{f}\right)$ if it is continuous on $I$, absolutely continuous on every compact subset of $] 0, T[, x(0)=\xi$, and for almost all $t \in I$, we have $x(t) \in D(B)$ and $\dot{x}(t) \in-B x(t)+f(t)$. A function $x: I \rightarrow X$ is called a weak solution of the Cauchy problem $\left(P_{f}\right)$ if there exist two sequence $\left\{f_{n}\right\}_{n \in N} \subset L^{1}(I, X)$ and $\left\{x_{n}\right\}_{n \in N} \subset C(I, X)$ such that $x_{n}$ is a strong solution of $\left(P_{f_{n}}\right), f_{n}$ converges to $f$ in $L^{1}(I, X)$, and $x_{n}$ converges to $x$ in $C(I, X)$.

It is known [4, Theorem 3.4] that for each $\xi \in \operatorname{cl} D(B)$ and $f \in L^{1}(I, X)$, there exists a unique weak solution $x_{f}(\cdot, \xi)$ of the Cauchy problem $\left(P_{f}\right)$. Moreover [4, Lemma 3.1] if $f, g \in L^{1}(I, X)$, and $x_{f}(\cdot, \xi), x_{g}(\cdot, \xi)$ are weak solutions of the Cauchy problems $\left(P_{f}\right),\left(P_{g}\right)$ then, for any $0 \leq t \leq T$, we have

$$
\left\|x_{f}(t, \xi)-x_{g}(t, \xi)\right\| \leq \int_{0}^{t}\|f(u)-g(u)\| d u .
$$

Set $X_{0}=\operatorname{cl} D(B)$. 
Remark 2.2. The map $\xi \rightarrow x_{f}(\cdot, \xi)$ is continuous from $X_{0}$ to $C(I, X)$. Indeed, since

$$
\begin{aligned}
& \frac{d}{d t}\left\|x_{f}\left(t, \xi_{1}\right)-x_{f}\left(t, \xi_{2}\right)\right\|^{2} \\
& \quad=2\left\langle\dot{x}_{f}\left(t, \xi_{1}\right)-f(t)+f(t)-\dot{x}_{f}\left(t, \xi_{2}\right), x_{f}\left(t, \xi_{1}\right)-x_{f}\left(t, \xi_{2}\right)\right\rangle \leq 0,
\end{aligned}
$$

we have $\left\|x_{f}\left(t, \xi_{1}\right)-x_{f}\left(t, \xi_{2}\right)\right\| \leq\left\|x_{f}\left(0, \xi_{1}\right)-x_{f}\left(0, \xi_{2}\right)\right\|=\left\|\xi_{1}-\xi_{2}\right\|$ for all $t \in I$, which implies the continuity of the map $\xi \rightarrow x_{f}(\cdot, \xi)$.

Now consider the Cauchy problem

$$
\dot{x}(t) \in-B x(t)+F(t, x(t)), \quad x(0)=\xi,
$$

where $F: I \times X \rightarrow 2^{X}$ satisfies $(\mathrm{H})$ and $\xi \in X_{0}$.

Definition 2.3. A function $x(\cdot, \xi): I \rightarrow X$ is called a weak solution of the Cauchy problem $\left(P_{\xi}\right)$ if there exists $f(\cdot, \xi) \in L^{1}(I, X)$, a selection of $F(\cdot, x(\cdot, \xi))$, such that $x(\cdot, \xi)$ is a weak solution of the Cauchy problem $\left(P_{f(\cdot, \xi)}\right)$.

We denote by $\mathscr{T}(\xi)$ the set of all weak solutions of $\left(P_{\xi}\right)$.

Theorem 2.4. Let $B$ be a maximal monotone map on $X$, and let $F: I \times X \rightarrow 2^{X}$ satisfy $(H)$. Then there exists a function $x(\cdot, \cdot): I \times X_{0} \rightarrow X$ such that

(i) $x(\cdot, \xi) \in \mathscr{T}(\xi)$ for every $\xi \in X_{0}$; and

(ii) $\xi \rightarrow x(\cdot, \xi)$ is continuous from $X_{0}$ to $C(I, X)$.

Proof. Fix $\varepsilon>0$ and set $\varepsilon_{n}=\varepsilon / 2^{n+1}, n \in N$. For $\xi \in X_{0}$, let $x_{0}(\cdot, \xi): I \rightarrow X$ be the unique weak solution of the Cauchy problem

$$
\dot{x}(t) \in-B x(t), \quad x(0)=\xi,
$$

and for $k$ and $\beta$ given by $\left(\mathrm{H}_{2}\right)$ and $\left(\mathrm{H}_{3}\right)$, define $\alpha: X_{0} \rightarrow L^{1}(I, R)$ by

$$
\alpha(\xi)(t)=\beta(t)+k(t)\left\|x_{0}(t, \xi)\right\| .
$$

Since, by Remark 2.2, the map $\xi \rightarrow x_{0}(\cdot, \xi)$ is continuous from $X_{0}$ to $C(I, X)$, from (2.2) it follows that $\alpha(\cdot)$ is continuous from $X_{0}$ to $L^{1}(I, R)$. Moreover, as consequence of $\left(\mathrm{H}_{2}\right)$ and $\left(\mathrm{H}_{3}\right)$, for each $\xi \in X_{0}$ we have:

$$
d\left(0, F\left(t, x_{0}(t, \xi)\right) \leq \alpha(\xi)(t) \text { a.e. in } I .\right.
$$

Define $G_{0}: X_{0} \rightarrow 2^{L^{1}(I, X)}$ and $H_{0}: X_{0} \rightarrow 2^{L^{1}(I, X)}$ by

$$
\begin{gathered}
G_{0}(\xi)=\left\{v \in L^{1}(I, X): v(t) \in F\left(t, x_{0}(t, \xi)\right) \text { a.e. } t \in I\right\}, \\
H_{0}(\xi)=\operatorname{cl}\left\{v \in G_{0}(\xi):\|v(t)\|<\alpha(\xi)(t)+\varepsilon_{0} \text { a.e. } t \in I\right\} .
\end{gathered}
$$

Clearly, by virtue of $(2.3)$ and Lemma $1.1, G_{0}(\cdot)$ is l.s.c. from $X_{0}$ into $\mathscr{D}$ and $H_{0}(\xi) \neq \varnothing$ for each $\xi \in X_{0}$. Hence, by Lemma 1.2 , there exists $h_{0}: X_{0} \rightarrow$ $L^{1}(I, X)$, a continuous selection of $H_{0}(\cdot)$. Set $f_{0}(t, \xi)=h_{0}(\xi)(t)$. Then 
$f_{0}(\cdot, \xi): X_{0} \rightarrow L^{1}(I, X)$ is continuous, $f_{0}(t, \xi) \in F\left(t, x_{0}(t, \xi)\right)$, and $\left\|f_{0}(t, \xi)\right\|$ $\leq \alpha(\xi)(t)+\varepsilon_{0}$ for $t \in I$ a.e.

Set $m(t)=\int_{0}^{t} k(u) d u$ and for $\xi \in X_{0}, n \geq 1$, define

$$
\beta_{n}(\xi)(t)=\int_{0}^{t} \alpha(\xi)(u) \frac{[m(t)-m(u)]^{n-1}}{(n-1) !} d u+T\left(\sum_{i=0}^{n} \varepsilon_{i}\right) \frac{[m(t)]^{n-1}}{(n-1) !}, \quad t \in I .
$$

Since $\alpha(\cdot)$ is continuous from $X_{0}$ to $L^{1}(I, R)$, by $(2.6)$ it follows that $\beta_{n}(\cdot)$ also is continuous from $X_{0}$ to $L^{1}(I, R)$.

Let $x_{1}(\cdot, \xi): I \rightarrow X$ be the unique weak solution of the Cauchy problem $\left(P_{f_{0}(\cdot, \xi)}\right)$. By $(2.1)$ we have

$$
\left\|x_{1}(t, \xi)-x_{0}(t, \xi)\right\| \leq \int_{0}^{t}\left\|f_{0}(u, \xi)\right\| d u \leq \int_{0}^{t} \alpha(\xi)(u) d u+\varepsilon_{0} T<\beta_{1}(\xi)(t)
$$

for each $\xi \in X_{0}$ and $t \in I \backslash\{0\}$.

We claim that there exist two sequences $\left\{f_{n}(\cdot, \xi)\right\}_{n \in N}$ and $\left.\left\{x_{n}(\cdot, \xi)\right\}\right)_{n \in N}$ such that for each $n \geq 1$, the following properties are satisfied:

(a) $\xi \rightarrow f_{n}(\cdot, \xi)$ is continuous from $X_{0}$ into $L^{1}(I, X)$;

(b) $f_{n}(t, \xi) \in F\left(t, x_{n}(t, \xi)\right)$ for each $\xi \in X_{0}$ and a.e. $t \in I$;

(c) $\left\|f_{n}(t, \xi)-f_{n-1}(t, \xi)\right\| \leq k(t) \beta_{n}(\xi)(t)$ for a.e. $t \in I$; and

(d) $x_{n}(\cdot, \xi)$ is the unique weak solution of the Cauchy problem $\left(P_{f_{n-1}(\cdot, \xi)}\right)$. Suppose we constructed $f_{1}, \ldots, f_{n}$ and $x_{1}, \ldots, x_{n}$ satisfying (a)-(d). Let $x_{n+1}(\cdot, \xi): I \rightarrow X$ be the unique weak solution of the Cauchy problem $\left(P_{f_{n}(\cdot, \xi)}\right)$. Then by (2.1) and (c) for $t \in I \backslash\{0\}$ we have

$$
\begin{aligned}
\left\|x_{n+1}(t, \xi)-x_{n}(t, \xi)\right\| \leq & \int_{0}^{t}\left\|f_{n}(u, \xi)-f_{n-1}(u, \xi)\right\| d u \\
\leq & \int_{0}^{t} k(u) \beta_{n}(\xi)(u) d u \\
= & \int_{0}^{t} \alpha(\xi)(u) \int_{u}^{t} k(\tau) \frac{[m(t)-m(\tau)]^{n-1}}{(n-1) !} d \tau d u \\
& +T\left(\sum_{i=0}^{n} \varepsilon_{i}\right) \int_{0}^{t} k(\tau) \frac{[m(\tau)]^{n-1}}{(n-1) !} d \tau \\
= & \int_{0}^{t} \alpha(\xi)(u) \frac{[m(t)-m(u)]^{n}}{n !} d u \\
& +T\left(\sum_{i=0}^{n} \varepsilon_{i}\right) \frac{[m(t)]^{n}}{n !}<\beta_{n+1}(\xi)(t) .
\end{aligned}
$$

Hence by $\left(\mathrm{H}_{2}\right)$,

$$
\begin{aligned}
d\left(f_{n}(t, \xi), F\left(t, x_{n+1}(t, \xi)\right)\right) & \leq k(t)\left\|x_{n+1}(t, \xi)-x_{n}(t, \xi)\right\| \\
& <k(t) \beta_{n+1}(\xi)(t) .
\end{aligned}
$$


By (2.8) and Lemma 1.1, we have that the multivalued map $G_{n+1}: X_{0} \rightarrow 2^{L^{1}(I, X)}$ defined by

$$
G_{n+1}(\xi)=\left\{v \in L^{1}(I, X): v(t) \in F\left(t, x_{n+1}(t, \xi)\right) \text { a.e. in } I\right\},
$$

is 1.s.c. with decomposable closed nonempty values, and by (2.8),

$$
H_{n+1}(\xi)=\operatorname{cl}\left\{v \in G_{n+1}(\xi):\left\|v(t)-f_{n}(t, \xi)\right\|<k(t) \beta_{n+1}(\xi)(t) \text { a.e. in } I\right\}
$$

is a nonempty set. Then by Lemma 1.2 , there exists $h_{n+1}: X_{0} \rightarrow L^{1}(I, X)$ a continuous selection of $H_{n+1}(\cdot)$. Setting $f_{n+1}(t, \xi)=h_{n+1}(\xi)(t)$ for $\xi \in X_{0}$, $t \in I$, we have that $f_{n+1}$ satisfies properties (a)-(c) of our claim.

By virtue of (c) and (2.7), we have

$$
\begin{aligned}
\left\|f_{n}(\cdot, \xi)-f_{n-1}(\cdot, \xi)\right\|_{1} & =\int_{0}^{T}\left\|f_{n}(u, \xi)-f_{n-1}(u, \xi)\right\| d u \\
& \leq \int_{0}^{T} \alpha(\xi)(u) \frac{[m(T)-m(u)]^{n}}{n !} d u+T\left(\sum_{i=0}^{n} \varepsilon_{i}\right) \frac{[m(T)]^{n}}{n !} \\
& \leq \frac{\left[\|k\|_{1}\right]^{n}}{n !}\left(\|\alpha(\xi)\|_{1}+T \varepsilon\right) .
\end{aligned}
$$

Since $\xi \rightarrow\|\alpha(\xi)\|_{1}$ is continuous it is locally bounded. Therefore (2.12) implies that for every $\xi \in X_{0}$ the sequence $\left(f_{n}\left(\cdot, \xi^{\prime}\right)\right)_{n \in N}$ satisfies the Cauchy condition uniformly with respect to $\xi^{\prime}$ on some neighborhood of $\xi$. Hence, if $f(\cdot, \xi)$ is the limit of $\left(f_{n}(\cdot, \xi)\right)_{n \in N}$, then $\xi \rightarrow f(\cdot, \xi)$ is continuous from $X_{0}$ into $L^{1}(I, X)$.

On the other hand, using (2.7) and (2.12), we have

$$
\left\|x_{n+1}(\cdot, \xi)-x_{n}(\cdot, \xi)\right\|_{\infty} \leq\left\|f_{n}(\cdot, \xi)-f_{n-1}(\cdot, \xi)\right\|_{1} \leq \frac{\left[\|k\|_{1}\right]^{n}}{n !}\left(\|\alpha(\xi)\|_{1}+T \varepsilon\right),
$$

and so as before, $\left(x_{n}(\cdot, \xi)\right)_{n \in N}$ is Cauchy in $C(I, X)$ locally uniformly with respect to $\xi$. Then, denoting its limit by $x(\cdot, \xi)$, it follows that the map $\xi \rightarrow$ $x(\cdot, \xi)$ is continuous from $X_{0}$ to $C(I, X)$. Since $x_{n}(\cdot, \xi)$ converges to $x(\cdot, \xi)$ uniformly and $d\left(f_{n}(t, \xi), F(t, x(t, \xi)) \leq k(t)\left\|x_{n}(t, \xi)-x(t, \xi)\right\|\right.$ passing to the limit along a subsequence $\left(f_{n_{k}}\right)_{k \in N}$ of $\left(f_{n}\right)_{n \in N}$ converging pointwise to $f$, we obtain that

$$
f(t, \xi) \in F(t, x(t, \xi)) \text { for each } \xi \in X_{0} \text { and } t \in I \text { a.e. }
$$

Let $x^{*}(\cdot, \xi)$ be the unique weak solution of the Cauchy problem

$$
\left(P_{f(\cdot, \xi)}\right) \quad \dot{x}(t) \in-B x(t)+f(t, \xi), \quad x(0)=\xi .
$$

By (2.1), we have

$$
\left\|x_{n+1}(t, \xi)-x^{*}(t, \xi)\right\| \leq \int_{0}^{t}\left\|f_{n}(u, \xi)-f(u, \xi)\right\| d u
$$


from which, letting $n \rightarrow \infty$, we get $x^{*}(\cdot, \xi) \equiv x(\cdot, \xi)$. Therefore $x(\cdot, \xi)$ is the weak solution of $\left(P_{f(\cdot, \xi)}\right)$, and by $(2.13)$, it follows that $x(\cdot, \xi) \in \mathscr{T}(\xi)$ for every $\xi \in X_{0}$.

\section{3. $A$ is the INFinitesimal Generator of a $C_{0}$-SEMigroup}

In this section $X$ is a separable Banach space and $\{G(t): t \geq 0\} \subset L(X, X)$ is a strongly continuous semigroup of bounded linear operators from $X$ to $X$ having infinitesimal generator $A$. Consider the Cauchy problem

$$
\dot{x}(t) \in A x(t)+F(t, x(t)), \quad x(0)=\xi,
$$

where $F: I \times X \rightarrow 2^{X}$ is a multivalued map satisfying $(\mathrm{H})$ and $\xi \in X$.

Definition 3.1. A function $x(\cdot, \xi): I \rightarrow X$ is called a mild solution of the Cauchy problem $\left(P_{\xi}\right)$ if there exists $f(\cdot, \xi) \in L^{1}(I, X)$ such that

(i) $f(t, \xi) \in F(t, x(t, \xi))$ for almost all $t \in I$; and

(ii) $x(t, \xi)=G(t) \xi+\int_{0}^{t} G(t-\tau) f(\tau, \xi) d \tau$ for each $t \in I$.

Remark 3.2. If $X$ is finite dimensional and $G(\cdot)$ is the identity, then every mild solution of $\left(P_{\xi}\right)$ is an absolutely continuous function satisfying

$$
\dot{x}(t, \xi) \in F(t, x(t, \xi)) \text { a.e. in } I, \quad x(0, \xi)=\xi .
$$

We denote by $\mathscr{T}(\xi)$ the set of all mild solutions of $\left(P_{\xi}\right)$.

Theorem 3.3. Let $A$ be the infinitesimal generator of a $C_{0}$-semigroup $\{G(t)$ : $t \geq 0\}$, and let $F$ satisfy $(H)$. Then there exists a function $x(\cdot, \cdot): I \times X \rightarrow X$ such that

(i) $x(\cdot, \xi) \in \mathscr{T}(\xi)$ for every $\xi \in X$; and

(ii) $\xi \rightarrow x(\cdot, \xi)$ is continuous from $X$ into $C(I, X)$.

Proof. Let $\varepsilon>0$ be fixed, and for $n \in N$, set $\varepsilon_{n}=\varepsilon / 2^{n+1}$. Let $M=$ $\sup \{\|G(t)\|: t \in I\}$, and for $\xi \in X$, define $x_{0}(\cdot, \xi): I \rightarrow X$ by $x_{0}(t, \xi)=$ $G(t) \xi$. Since

$$
\left\|x_{0}\left(t, \xi_{1}\right)-x_{0}\left(t, \xi_{2}\right)\right\| \leq\|G(t)\|\left\|\xi_{1}-\xi_{2}\right\| \leq M\left\|\xi_{1}-\xi_{2}\right\|,
$$

we have that $\xi \rightarrow x_{0}(\cdot, \xi)$ is continuous from $X$ to $C(I, X)$. For each $\xi \in X$, let $\alpha(\xi): I \rightarrow R$ be given by

$$
\alpha(\xi)(t)=\beta(t)+k(t)\left\|x_{0}(t, \xi)\right\| .
$$

Clearly $\alpha(\cdot)$ is continuous from $X$ to $L^{1}(I, R)$. Moreover, for each $\xi \in X$,

$$
d\left(0, F\left(t, x_{0}(t, \xi)\right)\right) \leq \alpha(\xi)(t) \text { for a.e. } t \in I .
$$

Let $G_{0}: X \rightarrow 2^{L^{1}(I, X)}$ and $H_{0}: X \rightarrow 2^{L^{1}(I, X)}$ be defined by (2.4) and (2.5) respectively. Then as in Theorem 2.4 one finds $h_{0}: X \rightarrow L^{1}(I, X)$, a continuous selection of $H_{0}(\cdot)$. Set $m(t)=\int_{0}^{t} k(\tau) d \tau$, and for $n \geq 1$, define 
$\beta_{n}: X \rightarrow L^{1}(I, R)$ by

$\beta_{n}(\xi)(t)=M^{n} \int_{0}^{t} \alpha(\xi)(u) \frac{[m(t)-m(u)]^{n-1}}{(n-1) !} d u+M^{n} T\left(\sum_{i=0}^{n} \varepsilon_{i}\right) \frac{[m(t)]^{n-1}}{(n-1) !}$,

$t \in I$.

Set $f_{0}(t, \xi)=h_{0}(\xi)(t)$, and define

$$
x_{1}(t, \xi)=G(t) \xi+\int_{0}^{t} G(t-\tau) f_{0}(\tau, \xi) d \tau, \quad t \in I .
$$

Then $f_{0}(t, \xi) \in F\left(t, x_{0}(t, \xi)\right),\left\|f_{0}(t, s)\right\| \leq \alpha(s)(t)+\varepsilon_{0}$, and for $t \in I \backslash\{0\}$,

$$
\begin{aligned}
\left\|x_{1}(t, \xi)-x_{0}(t, \xi)\right\| & \leq \int_{0}^{t}\|G(t-\tau)\|\left\|f_{0}(\tau, \xi)\right\| d \tau \leq M \int_{0}^{t}\left\|f_{0}(\tau, \xi)\right\| d \tau \\
& \leq M \int_{0}^{t} \alpha(\xi)(\tau) d \tau+M T \varepsilon_{0}<\beta_{1}(\xi)(t) .
\end{aligned}
$$

We claim that there exist two sequences $\left\{f_{n}(\cdot, \xi)\right\}_{n \in N}$ and $\left\{x_{n}(\cdot, \xi)\right\}_{n \in N}$ such that for each $n \geq 1$, the following properties are satisfied:

(a) $\xi \rightarrow f_{n}(\cdot, \xi)$ is continuous from $X$ into $L^{1}(I, X)$;

(b) $f_{n}(t, \xi) \in F\left(t, x_{n}(t, \xi)\right)$ for each $\xi \in X$ and a.e. $t \in I$;

(c) $\left\|f_{n}(t, \xi)-f_{n-1}(t, \xi)\right\| \leq k(t) \beta_{n}(\xi)(t)$ for a.e. $t \in I$; and

(d) $x_{n+1}(t, \xi)=G(t) \xi+\int_{0}^{t} G(t-\tau) f_{n}(\tau, \xi) d \tau$ for $t \in I$.

Suppose that we have already constructed $f_{1}, \ldots, f_{n}$ and $x_{1}, \ldots, x_{n}$ satisfying (a)-(d). Define $x_{n+1}(\cdot, \xi): I \rightarrow X$ by

$$
x_{n+1}(t, \xi)=G(t) \xi+\int_{0}^{t} G(t-\tau) f_{n}(\tau, \xi) d \tau, \quad t \in I .
$$

Then by (d), (c), for $t \in I \backslash\{0\}$, we have

$$
\begin{aligned}
& \left\|x_{n+1}(t, \xi)-x_{n}(t, \xi)\right\| \leq \int_{0}^{t}\|G(t-u)\|\left\|f_{n}(u, \xi)-f_{n-1}(u, \xi)\right\| d u \\
& \quad \leq M \int_{0}^{t}\left\|f_{n}(u, \xi)-f_{n-1}(u, \xi)\right\| d u \leq M \int_{0}^{t} k(u) \beta_{n}(\xi)(u) d u \\
& \quad=M^{n+1} \int_{0}^{t} \alpha(s)(\tau) \frac{[m(t)-m(\tau)]^{n}}{n !} d \tau+M^{n+1} T\left(\sum_{i=0}^{n} \varepsilon_{i}\right) \frac{[m(t)]^{n}}{n !} \\
& \quad<\beta_{n+1}(\xi)(t)
\end{aligned}
$$

and

$$
\begin{aligned}
d\left(f_{n}(t, \xi), F\left(t, x_{n+1}(t, \xi)\right)\right) & \leq k(t)\left\|x_{n+1}(t, \xi)-x_{n}(t, \xi)\right\| \\
& <k(t) \beta_{n+1}(\xi)(t) .
\end{aligned}
$$

Let $G_{n+1}: X \rightarrow 2^{L^{1}(I, X)}, H_{n+1}: X \rightarrow 2^{L^{1}(I, X)}$ be defined by (2.10), (2.11) respectively. By (3.4) and Lemma 1.1, $G_{n+1}(\cdot)$ is l.s.c. from $S$ into $\mathscr{D}$ and 
$H_{n+1}(\xi) \neq \varnothing$ for each $\xi \in X$. Hence by Lemma 1.2, there exists $h_{n+1}: X \rightarrow$ $L^{1}(I, X)$ a continuous selection of $H_{n+1}(\cdot)$. Then $f_{n+1}(t, \xi)=h_{n+1}(\xi)(t)$ satisfies the properties (a)-(c) of our claim. By (c) and (3.3), it follows that

$$
\begin{aligned}
\left\|x_{n+1}(\cdot, \xi)-x_{n}(\cdot, \xi)\right\|_{\infty} & \leq M\left\|f_{n}(\cdot, \xi)-f_{n-1}(\cdot, \xi)\right\|_{1} \\
& \leq \frac{\left[M\|k\|_{1}\right]^{n}}{n !}\left(M\|\alpha(\xi)\|_{1}+M T \varepsilon\right) .
\end{aligned}
$$

Therefore, $\left(f_{n}(\cdot, \xi)\right)_{n \in N}$ and $\left(x_{n}(\cdot, \xi)\right)_{n \in N}$ are Cauchy sequences in $L^{1}(I, X)$ and $C(I, X)$ respectively. Let $f(\cdot, \xi) \in L^{1}(I, X)$ and $x(\cdot, \xi) \in C(I, X)$ be their limits. Then as in the proof of Theorem 2.4 one can show: $\xi \rightarrow f(\cdot, \xi)$ is continuous from $X$ into $L^{1}(I, X), \xi \rightarrow x(\cdot, \xi)$ is continuous from $X$ into $C(I, X)$, and for all $\xi \in X$ and almost all $t \in I, f(t, \xi) \in F(t, x(t, \xi))$. Passing to the limit in (d) we obtain

$$
x(t, \xi)=G(t) \xi+\int_{0}^{t} G(t-\tau) f(\tau, \xi) d \tau \quad \text { for each } t \in I
$$

completing the proof.

\section{Some AdDitional Results}

In this section $X$ is a separable Banach space, assume that $F: I \times X \rightarrow 2^{X}$ satisfies $(\mathrm{H})$, and we consider the Cauchy problem

$$
\dot{x} \in F(t, x), \quad x(0)=\xi .
$$

We denote by $\mathscr{T}(\xi)$ the set of solutions of (4.1), i.e. of all $x \in A C(I, X)$ such that $x(0)=\xi$ and $\dot{x}(t) \in F(t, x(t))$ a.e. on $I$. It is known (see $[3,7])$ that there exists $r: X \rightarrow A C(I, X)$, a continuous selection of the multivalued map $\xi \rightarrow \mathscr{T}(\xi)$.

Proposition 4.1. Let $K$ be a nonempty compact convex subset of $X$ and assume that $\mathscr{T}(K)(T) \subset K$, where $\mathscr{T}(K)(T)=\{x(T): x \in \mathscr{T}(\xi), \xi \in K\}$. Then the boundary value problem

$$
\dot{x} \in F(t, x), \quad x(0)=x(T) \in K,
$$

admits a solution.

Proof. Let $\varphi: X \rightarrow X$ be given by $\varphi(\xi)=r(\xi)(T)$. Then $\varphi$ is continuous and $\varphi(K) \subset K$, hence by Schauder's Theorem there exists $\xi_{0} \in K$, a fixed point of $\varphi$. Then $r\left(\xi_{0}\right)(T)=\xi_{0}=r\left(\xi_{0}\right)(0)$ and so $x=r\left(\xi_{0}\right)$ is a solution of (4.2). 8].

Results of the type considered in Proposition 4.1 were also obtained in [1,

The following result is an analog of Michael's theorem in [12].

Theorem 4.2. If $Y$ is a closed nonempty subset of $X$, and $\varphi: Y \rightarrow A C(I, X)$ is a continuous map such that $\varphi(\xi) \in \mathscr{T}(\xi)$ for all $\xi \in Y$, then there exists 
$\varphi^{*}: X \rightarrow A C(I, X)$, a continuous extension of $\varphi$ such that $\varphi^{*}(\xi) \in \mathscr{T}(\xi)$ for all $\xi \in X$.

Proof. Let $\mathscr{T}^{\prime}(\xi)=\{\dot{x}: x \in \mathscr{T}(\xi)\}$ and set $\varphi^{\prime}(\xi)(t)=\frac{d}{d t} \varphi(\xi)(t)$. Then $\varphi^{\prime}: Y \rightarrow L^{1}(I, X)$ is continuous and satisfies $\varphi^{\prime}(\xi) \in \mathscr{T}^{\prime}(\xi)$ for all $\xi \in Y$. By Theorem 1 in [2] there exists $\lambda: X \rightarrow L^{1}(I, X)$, a continuous extension of $\varphi^{\prime}$, and by Theorem 2 in [3] there exists a continuous map $\psi: X \times L^{1}(I, X) \rightarrow$ $L^{1}(I, X)$ such that:

(i) $\psi(\xi, u) \in \mathscr{T}^{\prime}(\xi)$ for each $u \in L^{1}(I, X)$; and

(ii) $\psi(\xi, u)=u$ for each $u \in \mathscr{T}^{\prime}(\xi)$.

Define $\eta: X \rightarrow L^{1}(I, X)$ by $\eta(\xi)=\psi(\xi, \lambda(\xi))$. Then by (i), we obtain that $\eta(\xi) \in \mathscr{T}^{\prime}(\xi)$ for each $\xi \in X$. Moreover, since for $\xi \in Y$ we have $\lambda(\xi)=\varphi^{\prime}(\xi) \in \mathscr{T}^{\prime}(\xi)$, by (ii) it follows that for all $\xi \in Y$, we have $\eta(\xi)=$ $\psi\left(\xi, \varphi^{\prime}(\xi)\right)=\varphi^{\prime}(\xi)$. Therefore $\eta(\cdot)$ is a continuous extension of $\varphi^{\prime}(\cdot)$ and $\eta(\xi) \in \mathscr{T}^{\prime}(\xi)$ for each $\xi \in X$. Setting

$$
\varphi^{*}(\xi)(t)=\xi+\int_{0}^{t} \eta(\xi)(\tau) d \tau
$$

we obtain that $\varphi^{*}(\cdot)$ is a continuous extension of $\varphi(\cdot)$, and $\varphi^{*}(\xi) \in \mathscr{T}(\xi)$ for every $\xi \in X$.

Corollary 4.3. Let $\xi_{0}, \xi_{1} \in X, \xi_{0} \neq \xi_{1}$, and let $x_{0} \in \mathscr{T}\left(\xi_{0}\right), x_{1} \in \mathscr{T}\left(\xi_{1}\right)$. Then there exists a continuous map $\lambda:[0,1] \rightarrow A C(I, X)$ such that $\lambda(0)=x_{0}$, $\lambda(1)=x_{1}$, and for $\alpha \in[0,1], \lambda(\alpha) \in \mathscr{T}\left(\xi_{\alpha}\right)$, where $\xi_{\alpha}=(1-\alpha) \xi_{0}+\alpha \xi_{1}$.

Proof. Let $Y=\left\{\xi_{0}, \xi_{1}\right\}$ and $\varphi: Y \rightarrow A C(I, X)$ be given by $\varphi\left(\xi_{0}\right)=x_{0}$, $\varphi\left(\xi_{1}\right)=x_{1}$. By Theorem 4.2, there exists a continuous extension $\varphi^{*}(\cdot)$ of $\varphi(\cdot)$ such that $\varphi^{*}(\xi) \in \mathscr{T}(\xi)$ for every $\xi \in X$. Then the map $\lambda:[0,1] \rightarrow A C(I, X)$ defined by $\lambda(\alpha)=\varphi^{*}\left(\xi_{\alpha}\right)$, has the properties stated in the corollary.

\section{REFERENCES}

1. J. P. Aubin and A. Cellina, Differential inclusions, Springer-Verlag, Berlin, 1984.

2. A. Bressan and G. Colombo, Extensions and selections of maps with decomposable values, Studia Math. 90 (1988), 69-86.

3. A. Bressan, A. Cellina, and A. Fryszkowski, $A$ class of absolute retracts in spaces of integrable functions, Proc. Amer. Math. Soc. 384 (1991), 413-418.

4. H. Brezis, Operateurs maximaux monotones et semigroups de contractions dans les espaces de Hilbert, North-Holland, Amsterdam, 1973.

5. A. Cellina, On the set of solutions to Lipschitzian differential inclusions, Differential Integral Equations 1 (1988), 495-500.

6. A. Cellina and A. Ornelas, Representation of the attainable set for Lipschitzian differential inclusions, Rocky Mountain J. Math. (to appear).

7. R. M. Colombo, A. Fryszkowski, T. Rzezuchowski, and V. Staicu, Continuous selection of solutions sets of Lipschitzian differential inclusions, Funkcial. Ekvac. (to appear).

8. F. S. De Blasi and G. Pianigiani, Non-convex valued differential inclusions in Banach spaces, J. Math. Anal. Appl. (to appear). 
9. A. F. Filippov, Classical solutions of differential equations with multivalued right hand side, SIAM J. Control Optim. 5 (1967), 609-621.

10. H. Frankowska, A priori estimates for operational differential inclusions, J. Differential Equations 84 (1990), 100-128.

11. F. Hiai and $\mathrm{H}$. Umegaki, Integrals, conditions expectations and martingales of multivalued functions, J. Multivariate Anal. 7 (1971), 149-182.

12. E. Michael, Continuous selections. I, Ann. of Math. 63 (1956), 361-382.

13. A. A. Tolstonogov, On the properties of solutions of evolutions equations of subdifferential type in a Hilbert space, Proceedings of EQUADIFF 1989, Praha.

INTERnational School for Advanced Studies, Strada Costiera 11, 34014 Trieste, Italy 